\title{
The Role of Resilience and Gender in Relation to Infectious-Disease-Specific Health Literacy and Anxiety During the COVID-19 Pandemic
}

This article was published in the following Dove Press journal: Neuropsychiatric Disease and Treatment

\author{
Xueling Xiao' \\ Jinshun Xiao ${ }^{2}$ \\ Juqin Yao' \\ Yaling Chen ${ }^{3}$ \\ Leorey Saligan (1D ${ }^{4}$ \\ Nancy R Reynolds (D) \\ Honghong Wang (D) \\ 'Xiangya Nursing School of Central \\ South University, Changsha, Hunan, \\ People's Republic of China; ${ }^{2}$ School of \\ Information Engineering, Wuhan College, \\ Wuhan, Hubei, People's Republic of \\ China; ${ }^{3}$ Nursing Department, Medical \\ School of Wuhan University of Science \\ and Technology, Wuhan, Hubei, People's \\ Republic of China; ${ }^{4}$ National Institute of \\ Nursing Research/National Institute of \\ Health, Bethesda, MD, USA; ${ }^{5}$ Johns \\ Hopkins School of Nursing, Baltimore, \\ MD, USA
}

Background: Anxiety is one of the psychological problems being experienced by the general population during the current coronavirus disease 2019 (COVID-19) pandemic. Health literacy, such as infectious-disease-specific health literacy, is associated with anxiety, but the mechanism of such association is not clear. This study explored the relationship between infectious-disease-specific health literacy and anxiety.

Methods: We conducted an online survey in Hubei province, the early epicenter of the COVID-19 pandemic. A total of 1038 COVID-19-negative participants accomplished a battery of online survey tools determining their sociodemographic characteristics, perceived risk of acquiring COVID-19, infectious-disease-specific health literacy, resilience, and anxiety symptoms. We examined the moderated mediation models, in which the mediator was resilience and the moderator was gender.

Results: Most (63\%) of the study participants experienced at least mild anxiety. The indirect effect of infectious-disease-specific health literacy on anxiety through resilience was -0.282 (95\% confidence interval $[\mathrm{CI}]=[-0.511,-0.083])$, contributing to $32.7 \%$ of the total effect of health literacy on anxiety. Resilience fully mediated the association. The mediation effect of resilience tended to be stronger for the male participants than for the female participants as the moderation effect of gender remained until we included perceived risk of acquiring COVID-19 in the model.

Conclusion: Resilience was found to mediate the association between infectious-diseasespecific health literacy and anxiety. Individuals with good infectious-disease-specific health literacy are more likely to acquire higher resilience, which may in turn decrease their anxiety level. Males may benefit more from the mediation effect of resilience during the current pandemic. Infectious-disease-specific health literacy programs may help reduce the anxiety of the program participants by enhancing their resilience during a pandemic.

Keywords: health literacy, infectious-disease-specific health literacy, resilience, anxiety, pandemic, mental health

\section{Background}

The number of COVID-19 cases has climbed globally and has aroused worldwide attention. ${ }^{1}$ Various countries and organizations have adopted responses to COVID19, including quarantining different communities, social distancing, and closing of multiple institutions (schools, workforces, etc), which have induced a variety of abrupt changes in and disruptions of the daily lives of the public, many of which are inconvenient. $^{2,3}$ As a result, a psychological crisis is emerging among various groups, such as COVID-19 patients, healthcare providers, individuals with
Correspondence: Juqin Yao Xiangya Nursing School of Central South University, 172 Tongzipo Road, Yuelu District, Changsha, Hunan 4100I3,

People's Republic of China

$\mathrm{Tel} / \mathrm{Fax}+86-73 \mathrm{I}-2650266$

Email yaojq722@csu.edu.cn 
pre-existing conditions, and elderly people. They are at risk of developing mental diseases, and this risk extends to others as well. $^{2}$

Anxiety is a very common mental health problem during the current COVID-19 pandemic. ${ }^{4,5}$ Xiao et al found that anxiety is negatively associated with sleep quality among self-isolated people, ${ }^{6}$ and potentially deprives them of general immunity, which in turn can have a negative effect on their quality of life and ability to fight COVID-19 and other infections. ${ }^{7}$ Prior studies have indicated that the level of health literacy is negatively associated with anxiety. ${ }^{8-11}$ During the current COVID19 pandemic, health literacy has also been shown to be a protective factor for depression and a decrease in quality of life, ${ }^{12}$ and Drissi et $\mathrm{al}^{13}$ indicated that developing health literacy is an urgent need to prevent mental health problems among university students, but the strength of and the reason for the relationship between health literacy and mental health problems remain unclear. Therefore, we conducted this study to explore the anxiety level of the general population in Hubei, China, the early epicenter of COVID-19 in the early part of 2020 and one of the hardest hit by the pandemic. In addition, as COVID-19 is an emerging infectious disease, we examined infectiousdisease-specific health literacy ${ }^{14}$ and its mechanisms of action. We were especially interested in the roles of resilience and gender in the relationship between infectiousdisease-specific health literacy and anxiety.

People who are resilient tend to maintain a stable psychological state. Psychological resilience refers to the ability to maintain or quickly regain a healthy state after facing some adversity. ${ }^{15}$ If the individual's network is stable and resilient, the system will not enter a prolonged state of persistent symptom activation but will instead quickly recover from a stressful life event. In contrast, individuals who are not resilient are more prone to developing a mental disorder (eg, persistent anxiety) after suffering from a stressful life event. ${ }^{15}$ Empirically, the studies conducted during the severe acute respiratory syndrome (SARS) outbreak revealed that interventions targeting resilience improvement decreased anxiety. ${ }^{16,17}$ A systematic review also indicated that resilient adolescents experienced less anxiety. ${ }^{18}$ During the current COVID-19 epidemic, resilience has also been found to be negatively related to the level of anxiety. ${ }^{19-21}$ In this context, health literacy can be viewed as an asset that builds resilience at both the individual and community levels because people with health literacy are more likely to correctly receive and process information about diseases. ${ }^{22,23}$ Various studies have shown a positive relationship between health literacy and resilience, ${ }^{24-26}$ but few studies have explored the relationships between health literacy, resilience, and anxiety during the current COVID-19 pandemic. Therefore, based on these theoretical and empirical findings, we hypothesized that resilience mediates the relationship between infectious-disease-specific health literacy and anxiety.

In addition, from the genetic, hormonal, and environmental perspectives, gender may contribute to resilience. ${ }^{27}$ One study found that females may manifest greater fear of and difficulty in addressing the stressors encountered compared with males as the cognitive styles of males and females are different, which can potentially impact their resilience. ${ }^{28}$ In one study, a trigger event produced less anxiety for the males, and the decreased gray-matter volume in the males predicted greater resilience in them. ${ }^{29}$ As such, we included gender in our model, as shown in our study's conceptual framework (Figure 1), and hypothesized that gender moderates the indirect effect of infectious-disease-specific health literacy on anxiety.

In summary, in this study, we tested the mediating role of resilience and the moderating role of gender on the association between infectious-disease-specific health literacy and anxiety. As perceived risk of a disease also impacts psychological response ${ }^{30}$ and as it has been demonstrated as being associated with stress induced by COVID-19, ${ }^{31}$ such variable was controlled in our analysis.

This study was conducted in early March 2020, when the number of COVID-19 cases in Hubei province was still rising and a total lockdown was in place, which was similar to the situation in many other countries. This study can provide critical information for policymakers to understand the anxiety caused by the impact of COVID-19 and its associations with resilience and infectious-diseasespecific health literacy so that public health programs can

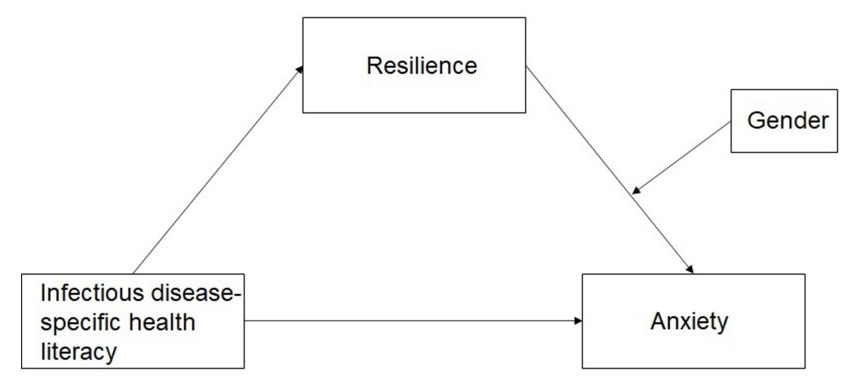

Figure I Conceptual framework. 
be developed to address issues related not just to this current pandemic but also to the future epidemics.

\section{Methods}

\section{Study Design, Participants, and Procedure}

This study was a cross-sectional survey conducted from March 3 to March 8, 2020, in Hubei province, China, which consists of 17 cities, including Wuhan. During the study period, the new cases of COVID-19 per day ranged from 36 to $134 .^{32}$ The eligible study participants were individuals who were 15 years or older and who were staying in Hubei province. Those who were diagnosed with COVID-19 were excluded from the study. The survey was performed via the online platform Wenjuanxing (https://www.wjx.cn/), and was set as accessible only to individuals whose internet protocol address was in Hubei.

We adopted snowball sampling to recruit participants for this study. First, 14 participants from different age groups (three aged over 50, three aged $40-50$, four aged $30-40$, two aged 20-30, one aged 19, and one aged 18) were invited to accomplish the survey. Second, the participants aged 18 or older were invited to share the survey link with others. Online informed consent or parental/guardian consent was obtained from each participant prior to the start of the survey. Two research assistants screened all the responses. The responses were deemed invalid if the participants chose the same option for more than 13 consecutive items in the survey (eg, the answers to all the items were the first option). A total of 1072 participants completed the survey, but 33 of them had invalid responses and one was found to be COVID-19 positive; these were excluded from the study, making the final sample 1038 participants covering all the 17 cities of Hubei province. It took the participants an average of 9.5 minutes to complete the survey, with a 7.43 standard deviation (SD).

\section{Measures}

We used a battery of instruments to measure the infectious-disease-specific health literacy, resilience, anxiety, perceived risk of acquiring COVID-19, and sociodemographic data of the study participants. The infectiousdisease-specific health literacy was assessed with a subscale of the surveillance survey questionnaire of health literacy among Chinese citizens developed by the National Health Commission of China. ${ }^{14}$ The subscale addresses the knowledge, attitude, and behavior related to infectious-disease prevention and control and consists of one true/false item, eight single-choice items, and three multiple-choice items. If the participants answered correctly, they are to obtain a score of 2 for each multiplechoice item and 1 for each of the other items. The total score ranges from 0 to 15 , and a score of 12 or above indicates good infectious-disease-specific health literacy. This subscale is reliable and is widely used in China. ${ }^{33,34}$ Its Cronbach's alpha in this study was 0.6.

Resilience was evaluated using the brief resilience scale developed by Smith et al $^{35}$ It has six items measuring the ability of the respondent to recover from stress. All the items are rated on the basis of a 5-point Likert scale, where 1 means strongly disagree and 5, strongly agree. Three of the items are reverse-coded. The final score for each respondent is the average of the scores for the six items, and the higher the score, the higher the resilience. This scale has been adopted in China and is widely used therein. $^{36}$ The reliability of this scale was acceptable in this study as the Cronbach's alpha was 0.7 .

Anxiety was measured with the Generalized Anxiety Disorder 7-item (GAD-7) scale. ${ }^{37}$ It has seven items assessing how frequently the respondent perceived the situation described in each item in the past two weeks. A 4-point Likert scale was adopted, as follows: $0=$ not at all; $1=$ several days; $2=$ more than a week; and $3=$ nearly every day. The total score ranges from 0 to 21 , and the higher the sum of scores, the higher the level of anxiety $(0-5=$ mild anxiety; $6-10=$ moderate anxiety; $11-15=$ severe anxiety). The Chinese version of GAD-7 is psychometrically favorable. ${ }^{38}$ Its Cronbach's alpha in this study was 0.9 .

The perceived risk of acquiring COVID-19 was assessed by four self-designed items, and a 5-point Likert scale was used to rate each item. The items were "What is the possibility that you will be infected by COVID-19?" (1 = very impossible; 5 = very possible), "How severe do you think the disease will be if you are infected by it?" $(1=$ very mild; 5 = very severe), "How afraid would you be of being quarantined if you were infected by COVID-19?" (1 = totally not afraid; 5 = very afraid), and "What is the possibility that your family will be infected by COVID19 ?" (1 = very impossible; 5 = very possible).

We also collected the study participants' sociodemographic data, including their ages, regions of residence, genders, education levels, marital statuses, employment statuses, monthly incomes, whether they had friends/ family who had been infected by COVID-19, whether there were people in their community who had been infected by COVID-19, and how they got information about COVID-19. 


\section{Data Analysis}

We used the SPSS 25.0 software to conduct statistical analysis. The mean (SD) and frequency were calculated to describe the quantitative and qualitative variables, respectively. To determine if infectious-disease-specific health literacy, resilience, and anxiety differed according to the various sociodemographic variables and perceived risk of acquiring COVID-19, we performed univariate analysis, including Pearson correlation test, Fisher's exact test, and analyses of variance.

For the mediation effect, Model 4 in SPSS PROCESS (the popular macro for SPSS, which is widely used to test for mediating and moderating ${ }^{39}$ ) was applied, and the indirect effect was evaluated through a bias-corrected bootstrapping test with 5000 bootstrap samples, in which the $95 \% \mathrm{CI}$ not including 0 indicates a significant indirect effect. As for the moderated mediation effect, we adopted linear regression and Model 14 in SPSS PROCESS. First, we used the linear regression model to explore the total effect of infectious-disease-specific health literacy on anxiety. Second, we computed the conditional indirect effect using the pick-a-point approach. Similarly, if the 95\% CI does not contain 0 , the conditional indirect effect is significant. To further explore the mediation and moderation effects, we first controlled for the sociodemographic variables, which were statistically significant in the univariate analysis, to adjust the models; then, we also added the statistically significant items of the perceived risk of acquiring COVID-19 as covariates.

\section{Results}

\section{Descriptive Statistics of the Sample and Correlation Between the Variables}

The average age of the study participants was 34.9 (SD = 13.16), with the ages ranging from 15 to 76 (see Table 1). More than half of the sample was female $(604,58.2 \%)$, and only 410 (39.5\%) scored 12 or higher, indicating good health literacy. The average scores for resilience and anxiety in the sample were 3.4 and $6.3(\mathrm{SD}=0.56$ and 5.03, respectively), and $63 \%$ (654) of the sample had at least mild anxiety, with $118(11.4 \%)$ having moderate anxiety and $75(7.2 \%)$, severe anxiety.

\section{Correlations Between the Variables}

As shown in Table 2, the study participants with good infectious-disease-specific health literacy showed greater resilience $(3.42 \pm 0.547$ vs $3.32 \pm 0.563)$ and lower anxiety
(5.77 \pm 4.586 vs $6.63 \pm 5.279)$ compared to those with low health literacy. The study participants' resilience was thus negatively related to their anxiety (Pearson coefficient $=$ -0.33 ). On the basis of the univariate analysis results listed in Table 1, the study participants' sociodemographic variables, including their age, region, gender, education level, marital status, employment status, monthly income, and television and Weibo as sources of information about COVID-19 were associated with their resilience and/or anxiety. All the four items for the perceived risk of acquiring COVID-19 were related to both resilience and anxiety.

\section{Mediation Effect of Resilience on the Relationship Between Infectious-Disease- Specific Health Literacy and Anxiety}

In the simple unadjusted mediation model shown in panel A of Figure 2, the total effect of infectious-disease-specific health literacy on anxiety (path $\mathrm{c}_{1}$ ) was -0.863 (path $\mathrm{c}_{1}$ : $95 \%$ $\mathrm{CI}=[-1.488,-0.238] ; \mathrm{p}=0.007)$. Infectious-disease-specific health literacy was thus positively associated with resilience (path $\mathrm{a}_{1}: \beta=0.097 ; 95 \% \mathrm{CI}=[0.027,0.166] ; \mathrm{p}=0.006$ ), and resilience was negatively related to anxiety (path $b_{1}: \beta=$ $-2.293 ; 95 \% \mathrm{CI}=[-3.443,-2.403] ; \mathrm{p}<0.001)$. The direct effect of infectious-disease-specific health literacy on anxiety trended toward significant (path $\mathrm{c}_{1}$ : $\beta=-0.581 ; 95 \% \mathrm{CI}=$ $[-1.175,0.013] ; \mathrm{p}=0.073)$, and the indirect effect was significant $(\beta=-0.282 ; 95 \% \mathrm{CI}=[-0.511,-0.083])$. That is, $32.7 \%$ of the effect of infectious-disease-specific health literacy on anxiety occurred indirectly through resilience.

In the adjusted model controlling for the significant sociodemographic variables except for gender (Figure 2, panel B), the indirect effect of health literacy on anxiety through resilience was slightly reduced but remained significant $(\beta=-0.261 ; 95 \% \mathrm{CI}=[-0.5,-0.036])$. As illustrated in panel $\mathrm{C}$ of Figure 2, after controlling for the four items for the perceived risk of acquiring COVID-19, the total effect of infectious-disease-specific health literacy on anxiety became insignificant, although the indirect effect was still significant $(\beta=-0.176 ; 95 \% \mathrm{CI}=[-0.362,-0.004])$.

\section{Mediation Effect of Resilience on the Relationship Between Infectious-Disease- Specific Health Literacy and Anxiety Moderated by Gender}

The results of the unadjusted, moderated mediation model (panel A of Figure 3) showed that the interaction between 
Table I Descriptive Characteristics of the Sample and Their Relationships with the Main Variables

\begin{tabular}{|c|c|c|c|}
\hline & Number (\%) or Mean (SD) & Resilience & Anxiety \\
\hline Age & $34.9 \pm 13.16$ & $3.4 \pm 0.56 * * *$ & $6.3 \pm 5.03 * * *$ \\
\hline \multicolumn{4}{|l|}{ Region } \\
\hline Urban & $524(50.5)$ & $3.4 \pm 0.56 * *$ & $6.3 \pm 4.91$ \\
\hline Rural & $514(49.5)$ & $3.3 \pm 0.55$ & $6.3 \pm 5.15$ \\
\hline \multicolumn{4}{|l|}{ Gender } \\
\hline Male & $434(4 I .8)$ & $3.4 \pm 0.57 * *$ & $6.2 \pm 5.40$ \\
\hline Female & $604(58.2)$ & $3.3 \pm 0.54$ & $6.3 \pm 4.75$ \\
\hline \multicolumn{4}{|l|}{ Education level } \\
\hline Primary school or lower & $62(6.0)$ & $3.3 \pm 0.57$ & $7.5 \pm 6.32^{* * *}$ \\
\hline Middle school & $338(32.6)$ & $3.4 \pm 0.54$ & $7.1 \pm 5.54$ \\
\hline High school & $230(22.2)$ & $3.3 \pm 0.61$ & $5.9 \pm 4.67$ \\
\hline Technical school or college & $206(19.8)$ & $3.4 \pm 0.54$ & $5.8 \pm 4.77$ \\
\hline Undergraduate or higher & $202(19.5)$ & $3.4 \pm 0.56$ & $5.3 \pm 4.02$ \\
\hline \multicolumn{4}{|l|}{ Marital status } \\
\hline Married & $396(38.2)$ & $3.3 \pm 0.58^{* * *}$ & $5.6 \pm 4.55^{* * *}$ \\
\hline Unmarried/divorced/widow & $642(61.8)$ & $3.4 \pm 0.54$ & $6.7 \pm 5.26$ \\
\hline \multicolumn{4}{|l|}{ Employment } \\
\hline Employed, with a stable job & $430(4 I .4)$ & $3.4 \pm 0.55^{* * *}$ & $6.3 \pm 5.02^{* * * *}$ \\
\hline Unemployed & $308(29.7)$ & $3.4 \pm 0.54$ & $7.1 \pm 5.48$ \\
\hline Student & $249(24.0)$ & $3.2 \pm 0.59$ & $5.1 \pm 3.86$ \\
\hline Retired & $51(4.9)$ & $3.4 \pm 0.52$ & $6.9 \pm 6.23$ \\
\hline \multicolumn{4}{|l|}{ Monthly income (yuan) } \\
\hline None & $360(34.7)$ & $3.3 \pm 0.57 * *$ & $5.9 \pm 4.5 I^{* *}$ \\
\hline $0-1000$ & $35(3.4)$ & $3.3 \pm 0.48$ & $7.5 \pm 5.89$ \\
\hline $100 \mid-2000$ & $113(10.9)$ & $3.4 \pm 0.54$ & $7.8 \pm 5.31$ \\
\hline $2001-3000$ & $155(14.9)$ & $3.4 \pm 0.54$ & $5.8 \pm 4.87$ \\
\hline $300 I-4000$ & $137(13.2)$ & $3.4 \pm 0.60$ & $6.4 \pm 5.24$ \\
\hline 4000 or above & $238(22.9)$ & $3.4 \pm 0.53$ & $6.3 \pm 5.35$ \\
\hline \multicolumn{4}{|c|}{ Has a friend or family who has been infected with COVID-I9 } \\
\hline Yes & $20(1.9)$ & $3.5 \pm 0.42$ & $8.0 \pm 4.39$ \\
\hline No & $989(95.3)$ & $3.4 \pm 0.56$ & $6.3 \pm 5.06$ \\
\hline Have no idea & $29(2.8)$ & $3.2 \pm 0.56$ & $5.6 \pm 4.15$ \\
\hline \multicolumn{4}{|c|}{ Has people in your community infected with COVID-19 } \\
\hline Yes & $256(24.7)$ & $3.4 \pm 0.58$ & $6.4 \pm 5.01$ \\
\hline No & $669(64.5)$ & $3.4 \pm 0.55$ & $6.2 \pm 5.13$ \\
\hline I suppose yes & $36(3.5)$ & $3.2 \pm 0.57$ & $7.8 \pm 5.04$ \\
\hline Have no idea & $77(7.4)$ & $3.3 \pm 0.52$ & $6.0 \pm 4.13$ \\
\hline \multicolumn{4}{|c|}{ Sources of information about COVID-19 } \\
\hline \multicolumn{4}{|l|}{ Television } \\
\hline No & $310(29.9)$ & $3.3 \pm 0.53^{*}$ & $6.6 \pm 4.83$ \\
\hline Yes & $728(70.1)$ & $3.4 \pm 0.57$ & $6.2 \pm 5.12$ \\
\hline \multicolumn{4}{|l|}{ WeChat } \\
\hline No & 393 (37.9) & $3.3 \pm 0.59$ & $6.5 \pm 5.24$ \\
\hline Yes & $645(62.1)$ & $3.4 \pm 0.54$ & $6.2 \pm 5.03$ \\
\hline
\end{tabular}

(Continued) 
Table I (Continued).

\begin{tabular}{|c|c|c|c|}
\hline & Number (\%) or Mean (SD) & Resilience & Anxiety \\
\hline \multicolumn{4}{|l|}{ News webpage } \\
\hline No & $22 \mid(2 \mid .3)$ & $3.4 \pm 0.53$ & $6.7 \pm 5.32$ \\
\hline Yes & $817(78.7)$ & $3.4 \pm 0.56$ & $6.2 \pm 4.95$ \\
\hline \multicolumn{4}{|l|}{ Weibo } \\
\hline No & $610(58.8)$ & $3.4 \pm 0.54$ & $6.6 \pm 5.16^{*}$ \\
\hline Yes & $428(4 I .2)$ & $3.4 \pm 0.59$ & $5.9 \pm 4.82$ \\
\hline \multicolumn{4}{|c|}{ Relatives or friends } \\
\hline No & $630(60.7)$ & $3.4 \pm 0.55$ & $6.4 \pm 5.19$ \\
\hline Yes & $408(39.3)$ & $3.3 \pm 0.57$ & $6.1 \pm 4.79$ \\
\hline \multicolumn{4}{|c|}{ Possibility of self-infection with COVID-19 } \\
\hline Very impossible & $310(29.9)$ & $3.5 \pm 0.62^{* * *}$ & $5.9 \pm 5.53 * * *$ \\
\hline Impossible & $479(46.1)$ & $3.4 \pm 0.52$ & $6.0 \pm 4.77$ \\
\hline Even & $173(16.7)$ & $3.2 \pm 0.52$ & $6.7 \pm 4.20$ \\
\hline Possible & $60(5.8)$ & $3.2 \pm 0.58$ & $8.4 \pm 5.38$ \\
\hline Very possible & $16(1.5)$ & $3.3 \pm 0.55$ & $10.4 \pm 6.02$ \\
\hline \multicolumn{4}{|c|}{ How serious if you are infected with COVID-19 } \\
\hline Very mild & $46(4.4)$ & $3.4 \pm 0.55^{*}$ & $4.0 \pm 5.26 * * *$ \\
\hline Mild & $69(6.6)$ & $3.5 \pm 0.49$ & $4.4 \pm 3.90$ \\
\hline Even & $130(12.5)$ & $3.4 \pm 0.56$ & $5.6 \pm 4.48$ \\
\hline Severe & $360(34.7)$ & $3.3 \pm 0.57$ & $6.1 \pm 4.36$ \\
\hline Very severe & $433(4 I .7)$ & $3.4 \pm 0.56$ & $7.2 \pm 5.61$ \\
\hline \multicolumn{4}{|c|}{ Will you be afraid of being quarantined if you are infected with COVID- I9? } \\
\hline Totally not afraid & $134(12.9)$ & $3.7 \pm 0.66 * * *$ & $4.3 \pm 5.24 * * *$ \\
\hline Not afraid & $259(25.0)$ & $3.4 \pm 0.52$ & $5.1 \pm 3.91$ \\
\hline Even & $112(10.8)$ & $3.3 \pm 0.49$ & $5.7 \pm 4.63$ \\
\hline Afraid & $387(37.3)$ & $3.3 \pm 0.49$ & $6.5 \pm 4.33$ \\
\hline Very afraid & $146(14.1)$ & $3.2 \pm 0.60$ & $10.0 \pm 6.48$ \\
\hline \multicolumn{4}{|c|}{ Possibility of a family member being infected with COVID-19 } \\
\hline Very impossible & $290(27.9)$ & $3.4 \pm 0.60 * * *$ & $5.9 \pm 5.70 * * *$ \\
\hline Impossible & $474(45.7)$ & $3.4 \pm 0.53$ & $5.8 \pm 4.56$ \\
\hline Even & I8I (17.4) & $3.2 \pm 0.51$ & $6.8 \pm 4.67$ \\
\hline Possible & $78(7.5)$ & $3.2 \pm 0.56$ & $9.2 \pm 5.31$ \\
\hline Very possible & $15(1.4)$ & $3.5 \pm 0.66$ & $7.3 \pm 5.52$ \\
\hline
\end{tabular}

Notes: $*<0.05 ; * *<0.01$; $* * *<0.001$

Abbreviation: SD, standard deviation.

gender and resilience was related to anxiety $(\beta=1.222$; $95 \% \mathrm{CI}=[0.175,2.269] ; \mathrm{p}=0.022)$, suggesting that gender could moderate the indirect effect of infectiousdisease-specific health literacy on anxiety. To further understand the moderating effect of gender, the conditional indirect effect was estimated for both the male and female participants. The results showed a $-0.35(95 \% \mathrm{CI}=$ $[-0.629,-0.104])$ coefficient for the male participants and $-0.232(95 \% \mathrm{CI}=[-0.421,-0.069])$ for the female participants. This indicated that the indirect effect turned stronger among the male participants; this pattern is visualized in panel A of Figure 4. The visual unparallel lines show that among the participants with lower resilience, the model estimated stronger justification for anxiety among the male participants than among the female participants. Among the study participants with higher resilience, the opposite was evident.

In panel B of Figure 3, after adjusting the model by controlling for the sociodemographic covariates, the indirect effect of infectious-disease-specific health literacy on 
Table 2 Relationships Between Infectious-Disease-Specific Health Literacy, Resilience, and Anxiety

\begin{tabular}{|l|l|l|l|}
\hline Variables & I & $\mathbf{2}$ & $\mathbf{3}$ \\
\hline $\begin{array}{l}\text { I. Infectious-disease-specific health } \\
\text { literacy }\end{array}$ & $\mathrm{I}$ & & \\
2. Resilience & $7.463^{* * a}$ & $\mathrm{I}$ & \\
3. Anxiety & $7.433^{* * a}$ & $0.329 * * * \mathrm{~b}$ & $\mathrm{I}$ \\
\hline
\end{tabular}

Notes: ${ }^{a}$ Value of $F$ in the analysis of variance; ${ }^{b}$ Value of $r$ in the Pearson correlation test; $* *<0.01$, *** $<0.001$.

anxiety remained, although it was slightly reduced. That is, the effect among the male participants was -0.305 $(95 \% \mathrm{CI}=[-0.587,-0.047])$ and that among the female participants was $-0.227(95 \% \mathrm{CI}=[-0.441,-0.034])$ (panel B, Figure 4). The moderating effect, however, was no longer significant.

After controlling for both the sociodemographic covariates and the perceived risk of acquiring COVID-19, the total effect of infectious-diseasespecific health literacy on anxiety became insignificant, as can be seen in panel $\mathrm{C}$ of Figure 3. The interaction between gender and resilience was also not significant. The indirect effect, however, remained significant: the effect for the male participants was $-0.197(95 \% \mathrm{CI}=$ $[-0.422,-0.003])$ and that for the female participants was $-0.163 \quad\left(95 \% \quad \mathrm{CI}=\left[\begin{array}{ll}-0.338, & -0.002\end{array}\right]\right.$. Panel C (Figure 4) suggests that the anxiety gap between the male and female participants was reduced as the resilience increased.

\section{Discussion}

In this study, we found that during the current COVID-19 pandemic, the majority of the non-infected individuals from Hubei province in China experienced at least mild anxiety. In addition, a moderated mediation model including resilience as the mediator and gender as the moderator offered an understanding of the association between infectious-disease-specific health literacy and anxiety. The study findings revealed that the rate of high anxiety among the study participants $(18.6 \%$ with moderate to severe anxiety) was similar to that among the community and health professionals in Wuhan province $(23.84 \%),{ }^{40}$ and the prevalence in our sample was far more than a pooled rate of $2.45 \%$ in mainland China. ${ }^{41}$

The main finding in this study was the indirect effect of infectious-disease-specific health literacy on anxiety through resilience. Health literacy was associated with increased resilience, which in turn was associated with a decreased level of anxiety. One potential reason for this was that the individuals with good infectious-diseasespecific health literacy were able to acquire correct information about COVID-19 and process this critically when faced with a large amount of complex and contradictory information, which made them resilient to infodemic. ${ }^{42}$
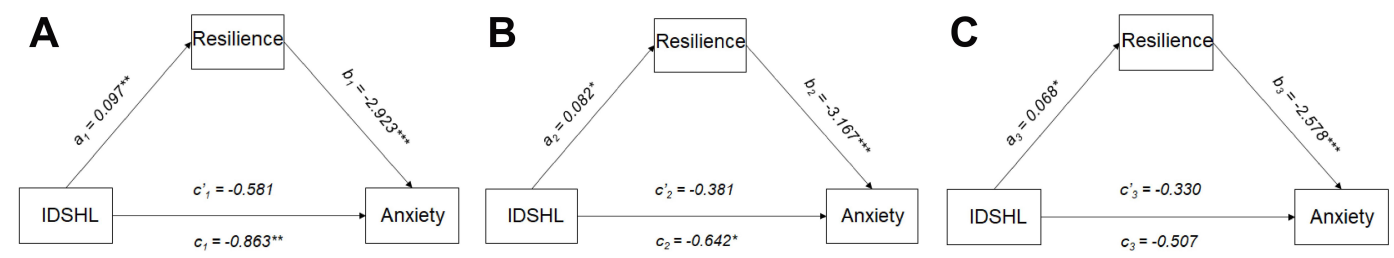

Figure 2 Mediation model.

Notes: (A) Unadjusted model. (B) Adjusted model controlled for significant sociodemographic variables. (C) Adjusted model controlled for both significant sociodemographic variables and the four items for the perceived risk of acquiring COVID-19. ${ }^{*} p<0.05 ; * * p<0.01$; ***p $<0.001$.

Abbreviation: IDSHL, infectious-disease-specific health literacy.
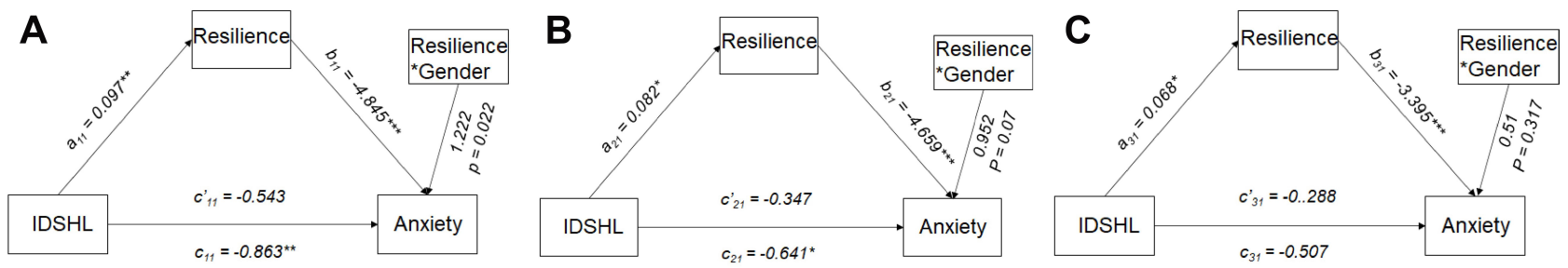

Figure 3 Moderated mediation model.

Notes: (A) Unadjusted model. (B) Adjusted model controlled for significant sociodemographic variables. (C) Adjusted model controlled for both significant sociodemographic variables and the four items for the perceived risk of acquiring COVID-I9. *p $<0.05$; **p $<0.01$; ***p $<0.001$.

Abbreviation: IDSHL, infectious-disease-specific health literacy. 

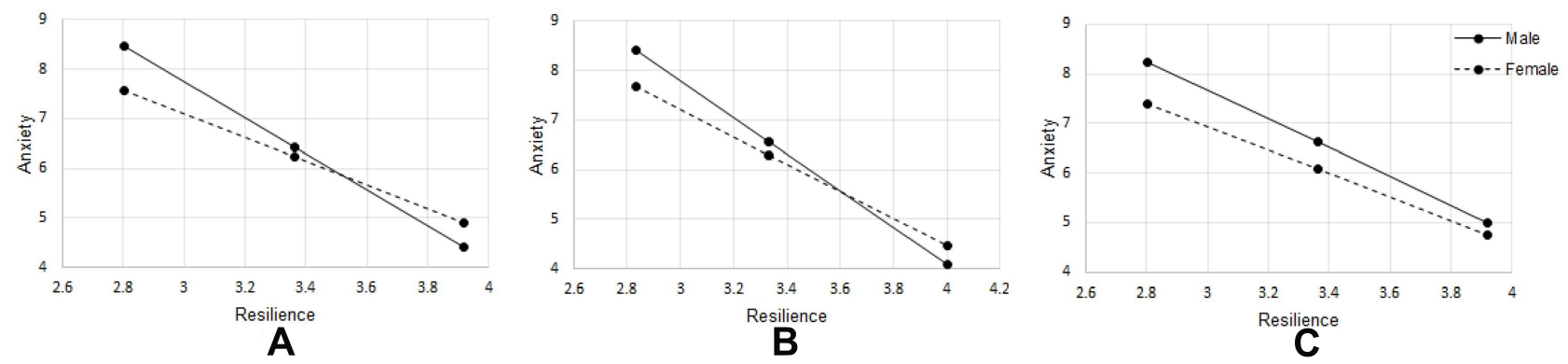

Figure 4 Visualized moderation effect of gender.

Notes: (A) Unadjusted model. (B) Adjusted model controlled for significant sociodemographic variables. (C) Adjusted model controlled for both significant sociodemographic variables and the four items for the perceived risk of acquiring COVID-19.

Besides, infectious-disease-specific health literacy helped the study participants understand the reasons behind the institutional preventive strategies and guidelines, which might have facilitated their compliance with these and might have simultaneously enabled them to recover from the negative impact of COVID-19. ${ }^{43}$ The finding regarding resilience is consistent with that of the previous studies that have been conducted during the current pandemic: ${ }^{44-46}$ that various COVID-19-negative populations with a higher level of resilience experienced less anxiety. Most of the previous studies on resilience and anxiety simply focused on the association between the two and did not consider the role of infectious-diseasespecific health literacy even though the importance of such was highlighted during the current epidemic to enhance the people's resilience. ${ }^{47}$ Unfortunately, only a small part of the sample had good infectious-disease-specific health literacy, indicating the need to develop programs to improve the infectious-disease-specific health literacy of such population. Infectious-disease-specific health literacy programs are vital for individuals to adopt behaviors that can protect themselves and can prevent them from spreading the infection. ${ }^{43}$

The study results revealed that the mediating role of resilience on the relationship between infectious-diseasespecific health literacy and anxiety was potentially different between the male and female study participants, showing a stronger mediation effect of resilience among the male participants than among the female participants. Prior studies indicated that women are more likely to perceive more distress from and focus on the negative aspects of a stressful event, ${ }^{48,49}$ a phenomenon that has been shown to be consistent during the current COVID-19 pandemic as COVID-19 has so far shown a greater psychological impact on females than on males. ${ }^{50}$ These different levels of vulnerability to a stressor may contribute to the gender difference in the model. Interestingly, the moderating effect of gender became insignificant only when we included the perceived risk of acquiring COVID19 in the conditional mediation model. The female participants had a significantly higher perceived risk of acquiring COVID-19 than the male participants, ${ }^{51}$ which may have buffered the moderating effect of gender in this model because risk perception is highly associated with psychological responses. ${ }^{52}$ The gender-specific pathways cast light on the need to develop gender-specific interventions to reduce anxiety. Especially, when the resilience was at a low level, the female participants suffered much more than the male participants did from the impact of COVID19. The future interventions should take this gap into account.

The perceived risk of acquiring COVID-19 may play an important role in the relationships between infectiousdisease-specific health literacy, resilience, and anxiety as the total effect became insignificant when it was included in the tested models. Infectious-disease-specific health literacy impacts how people interpret the bodily sensations or changes during an epidemic, and if people perceive their bodily changes as being indicative of COVID-19, they may experience health anxiety. ${ }^{53}$ The level of people's health anxiety has been elevated during the current COVID-19 pandemic, ${ }^{54}$ and this contributes to the emergence of general anxiety symptoms. ${ }^{50}$ Also, a recent study indicated that the participants' reported risk of acquiring COVID-19 was related to their functional fear, which has a conceptual similarity with health anxiety, as stated by Harper et al. ${ }^{55}$ The potential interaction between perceived risk of acquiring COVID-19 and health anxiety may explain the change after controlling for reported risk in the models. Studies taking the perceived risk of acquiring 
COVID-19 and health anxiety into account should be conducted to provide evidence of how to reduce anxiety among the general population during an epidemic.

As this study was conducted in an epicenter during the peak period of the COVID-19 pandemic in China, it provides valuable implications for public health. Besides, as this study was conducted through an online platform and the responses of the study participants were obtained very quickly, this suggests that specific interventions administered through online platforms may be feasible. Considering the importance of health literacy as associated with anxiety through resilience, infectious-disease-specific health literacy programs may help enhance the resilience and reduce the anxiety of the general population. Specifically, how to help people obtain the right information and develop the needed skills to protect themselves from COVID-19 is vital when developing intervention strategies. Except of course for the actions to be taken to control an epidemic, it is much more crucial to plan approaches to improving the public awareness about an infectious disease in advance so that the public can become more resilient to the next pandemic. In addition, the gender-specific vulnerabilities to the pandemic should be addressed. Further studies using other validated measures to confirm or refine the relationships between resilience, infectious-disease-specific health literacy, and anxiety are warranted.

This study had several limitations. First, as the study was a cross-sectional survey, the true causal relationships between the variables could not be verified. Second, we recruited participants based on an online platform, which may have led to selection bias, for which reason the result generalization should be exercised with caution. This notwithstanding, our sample covered all the 17 cities in Hubei province, and the demographic data showed variation in the sample. In addition, the survey relied on self-reporting, and we of course were not able to gather data from our sample regarding their anxiety level before the start of the COVID-19 pandemic. Further longitudinal studies adopting audio call or other approaches to interviewing the study participants are thus warranted.

To the best of our knowledge, this was the first study that explored the relationships between infectious-diseasespecific health literacy, resilience, and anxiety during the current COVID-19 pandemic. We found in this study that the majority of the general population in the initial epicenter of COVID-19, Hubei province in China, experienced at least mild anxiety. It is important to address these psychological issues to restore the public health and to better plan for future pandemics. Enhancing the resilience of the public is important to reduce their anxiety, based on the relationship between infectious-diseasespecific health literacy and anxiety observed in this study. More importantly, the results of this study highlight the importance of health literacy regarding infectious disease: efficient gender-specific approaches should be provided to improve people's health literacy and reduce their anxiety during a pandemic.

\section{Data Sharing Statement}

The study data are not openly available. Bona fide researchers interested in the related topic can get in touch with our corresponding author (Juqin Yao) for the details of the study data and how to access them.

\section{Ethics}

This study was approved by the Ethics Committee of the School of Nursing of the Central South University. We carried out all the procedures as per the Declaration of Helsinki. Online informed consent was obtained from all the study participants. We first asked the participants to report their ages to us. If a prospective participant indicated an age under 18 years old, a message showed up asking the participant to consult with a parent/guardian for participation approval. After the parent/guardian read the information and ticked a box showing his or her assent, the participant could provide online informed consent and join the survey. For the prospective participants 18 years old or above, they provided their online informed consent by ticking a box after reading the information about the study to indicate that they agree to join the study.

\section{Acknowledgment}

We would like to express our sincere appreciation to all the study participants for their cooperation in this survey.

\section{Funding}

This study was funded by the Innovation-Driven Project of the Central South University (Grant No. 2018zzts244).

\section{Disclosure}

The authors report no conflicts of interest for this work.

\section{References}

1. World Health Organization [homepage on the Internet]. Coronavirus (COVID-19); 2020. Available from: https://covid19.who.int/. Accessed April 30, 2020. 
2. The Lancet Psychiatry. Isolation and inclusion. Lancet Psychiatry. 2020;7(5):371. doi:10.1016/S2215-0366(20)30156-5

3. Araujo FJO, Lima LS, Cidade PIM, Nobre CB, Neto MLR. Impact of Sars-Cov-2 and its reverberation in global higher education and mental health. Psychiatry Res. 2020;288:112977. doi:10.1016/j. psychres.2020.112977

4. Wang C, Pan R, Wan X, et al. Immediate psychological responses and associated factors during the initial stage of the 2019 coronavirus disease (COVID-19) epidemic among the general population in China. Int J Environ Res Public Health. 2020;17(5).

5. Salari N, Hosseinian-Far A, Jalali R, et al. Prevalence of stress, anxiety, depression among the general population during the COVID-19 pandemic: a systematic review and meta-analysis. Global Health. 2020;16(1):57. doi:10.1186/s12992-020-00589-w

6. Xiao H, Zhang Y, Kong D, Li S, Yang N. The effects of social support on sleep quality of medical staff treating patients with coronavirus disease 2019 (COVID-19) in january and february 2020 in China. Med Sci Monit. 2020;26:e923549. doi:10.12659/ MSM.923921

7. Shneider A, Kudriavtsev A, Vakhrusheva A. Can melatonin reduce the severity of COVID-19 pandemic? Int Rev Immunol. 2020;1-10.

8. Palazzo MC, Dell'Osso B, Altamura AC, Stein DJ, Baldwin DS. Health literacy and the pharmacological treatment of anxiety disorders: a systematic review. Int J Environ Res Public Health. 2014;29 (3):211-215.

9. Oh HJ, Lee H. When do people verify and share health rumors on social media? The effects of message importance, health anxiety, and health literacy. $J$ Health Commun. 2019;24(11):837-847.

10. Halbach SM, Enders A, Kowalski C, et al. Health literacy and fear of cancer progression in elderly women newly diagnosed with breast cancer: a longitudinal analysis. Patient Educ Couns. 2016;99 (5):855-862. doi:10.1016/j.pec.2015.12.012

11. Ghisi GLM, Chaves G, Britto RR, Oh P. Health literacy and coronary artery disease: a systematic review. Patient Educ Couns. 2018;101 (2):177-184. doi:10.1016/j.pec.2017.09.002

12. Nguyen HC, Nguyen MH, Do BN, et al. People with suspected COVID-19 symptoms were more likely depressed and had lower health-related quality of life: the potential benefit of health literacy. J Clin Med. 2020;9(4):965. doi:10.3390/jcm9040965

13. Drissi N, Alhmoudi A, Al Nuaimi H, Alkhyeli M, Alsalami S, Ouhbi S. Investigating the impact of COVID-19 lockdown on the psychological health of university students and their attitudes toward mobile mental health solutions: two-part questionnaire study. JMIR Form Res. 2020;4(10):e19876. doi:10.2196/19876

14. Nie X. Statistic analysis of 2012 Chinese residents health literacy monitoring. Chin J Health Educ. 2014;30(2):178-181.

15. Borsboom D. A network theory of mental disorders. World Psychiatry. 2017;16(1):5-13. doi:10.1002/wps.20375

16. Ng SM, Chan TH, Chan CL, et al. Group debriefing for people with chronic diseases during the SARS pandemic: strength-focused and meaning-oriented approach for resilience and transformation (SMART). Community Ment Health J. 2006;42(1):53-63. doi:10.10 07/s10597-005-9002-y

17. Chan CL, Chan TH, Ng SM. The strength-focused and meaning-oriented approach to resilience and transformation (SMART): a body-mind-spirit approach to trauma management. Soc Work Health Care. 2006;43(2-3):9-36. doi:10.1300/J010v43n02 03

18. Schäfer J, Naumann E, Holmes EA, Tuschen-Caffier B, Samson AC. Emotion regulation strategies in depressive and anxiety symptoms in youth: a meta-analytic review. $J$ Youth Adolesc. 2017;46(2):261-276. doi:10.1007/s10964-016-0585-0

19. Luceño-Moreno L, Talavera-Velasco B, García-Albuerne Y, Martín-García J. Symptoms of posttraumatic stress, anxiety, depression, levels of resilience and burnout in Spanish health personnel during the COVID-19 pandemic. Int J Environ Res Public Health. 2020;17(15):5514. doi:10.3390/ijerph17155514
20. Savitsky B, Findling Y, Ereli A, Hendel T. Anxiety and coping strategies among nursing students during the COVID-19 pandemic. Nurse Educ Pract. 2020;46:102809. doi:10.1016/j.nepr.2020.102809

21. Liu CH, Zhang E, Wong GTF, Hyun S, Hahm HC. Factors associated with depression, anxiety, and PTSD symptomatology during the COVID-19 pandemic: clinical implications for U.S. young adult mental health. Psychiatry Res. 2020;290:113172. doi:10.1016/j. psychres.2020.113172

22. Chandra A, Joie DA, Howard S, et al. Building Community Resilience to Disasters: A Way Forward to Enhance National Health Security. Santa Monica (CA): RAND Corporation; 2011.

23. Kickbusch I, Pelikan JM, Apfel F, Tsouros A. Health Literacy. WHO Regional Office for Europe; 2013.

24. Seifi B, Ghanizadeh G, Seyedin H. Disaster health literacy of middle-aged women. J Menopausal Med. 2018;24(3):150-154. doi: $10.6118 / \mathrm{jmm} .2018 .24 .3 .150$

25. Jacobs RJ, Ownby RL, Acevedo A, Waldrop-Valverde D. A qualitative study examining health literacy and chronic illness self-management in Hispanic and non-Hispanic older adults. $J$ Multidiscip Healthc. 2017;10:167-177. doi:10.2147/JMDH. S135370

26. Fund N, Ash N, Porath A, Shalev V, Koren G. Comparison of mortality and comorbidity rates between Holocaust survivors and individuals in the general population in Israel. JAMA Netw Open. 2019;2(1):e186643. doi:10.1001/jamanetworkopen.2018.6643

27. Szanton SL, Gill JM. Facilitating resilience using a society-to-cells framework: a theory of nursing essentials applied to research and practice. ANS Adv Nurs Sci. 2010;33(4):329-343. doi:10.1097/ ANS.0b013e 3181fb2ea2

28. Hodes GE, Epperson CN. Sex differences in vulnerability and resilience to stress across the life span. Biol Psychiatry. 2019;86 (6):421-432. doi:10.1016/j.biopsych.2019.04.028

29. Goldstein-Piekarski AN, Greer SM, Saletin JM, Harvey AG, Williams LM, Walker MP. Sex, sleep deprivation, and the anxious brain. $J$ Cogn Neurosci. 2018;30(4):565-578. doi:10.1162/ jocn_a_01225

30. Lazarus RS, Folkman S. Stress, Appraisal, and Coping. Springer Publishing Company: New York; 1984.

31. Qian M, Wu Q, Wu P, et al. Psychological responses, behavioral changes and public perceptions during the early phase of the COVID-19 outbreak in China: a population based cross-sectional survey. medRxiv. 2020.

32. CDCC. The distribution of COVID-19 epidemic; 2020. Available from: http://2019ncov.chinacdc.cn/2019-nCoV/. Accessed April 23, 2020.

33. Huang X, Li C, Li Y, Li L, Nie X. Analysis of status and influence factors of health literacy of infectious diseases prevention of the urban and rural residents in China. Chin J Health Educ. 2015;31(2).

34. Wu Y, Nan H, Tie Y, Guo J. Analysis of status and influencing factors of health literacy of infection disease prevention and control among residents in Shaanxi province. Infect Dis Info. 2019;32 (5):407-410.

35. Smith BW, Dalen J, Wiggins K, Tooley E, Christopher P, Bernard J. The brief resilience scale: assessing the ability to bounce back. Int J Behav Med. 2008;15(3):194-200. doi:10.1080/10705500802 222972

36. Chen W, Liu J, Luo J, Guoqing L. Reliability and validity of the Chinese version of brief resilience scale. Chin J Clin Psychol. 2020;28(1):24-28.

37. Spitzer RL, Kroenke K, Williams JB, Lowe B. A brief measure for assessing generalized anxiety disorder: the GAD-7. Arch Intern Med. 2006;166(10):1092-1097. doi:10.1001/archinte.166.10.1092

38. Zeng Q, He Y, Liu H, et al. Reliability and validity of Chinese version of the generalized anxiety disorder 7-item (GAD-7) scale in screening anxiety disorders in outpatients from traditional Chinese internal department. Chin Ment Health J. 2013;27(3):163-168. 
39. Hayes AF. Introduction to Mediation, Moderation, and Conditional Process Analysis: A Regression-Based Approach. 2nd ed. New York: Guilford Press; 2018.

40. Ni MY, Yang L, Leung CMC. Mental health, risk factors, and social media use during the COVID-19 epidemic and cordon sanitaire among the community and health professionals in Wuhan, China: cross-sectional survey. JMIR Ment Health. 2020;7(5):e19009. doi:10.2196/19009

41. Guo X, Meng Z, Huang G, et al. Meta-analysis of the prevalence of anxiety disorders in mainland China from 2000 to 2015. Sci Rep. 2016;6(1):28033. doi:10.1038/srep28033

42. Zarocostas J. How to fight an infodemic. Lancet. 2020;395 (10225):676. doi:10.1016/S0140-6736(20)30461-X

43. Paakkari L, Okan O. COVID-19: health literacy is an underestimated problem. Lancet Public Health. 2020;5(5):e249-e250. doi:10.1016/ S2468-2667(20)30086-4

44. Kimhi S, Eshel Y, Marciano H, Adini B. Distress and resilience in the days of COVID-19: comparing two ethnicities. Int $J$ Environ Res Public Health. 2020;17(11):3956. doi:10.3390/ijerph17113956

45. Fegert JM, Vitiello B, Plener PL, Clemens V. Challenges and burden of the coronavirus 2019 (COVID-19) pandemic for child and adolescent mental health: a narrative review to highlight clinical and research needs in the acute phase and the long return to normality. Child Adolesc Psychiatry Ment Health. 2020;14(1):20. doi:10.1186/ s13034-020-00329-3

46. Bohlken J, Schömig F, Lemke MR, Pumberger M, Riedel-Heller SG. COVID-19 pandemic: stress experience of healthcare workers: a short current review. Psychiatr Prax. 2020;47(4):190-197.

47. Ratzan SC, Sommariva S, Rauh L. Enhancing global health communication during a crisis: lessons from the COVID-19 pandemic. Public Health Res Pract. 2020;30(2). doi:10.17061/phrp3022010
48. Dévieux JG, Malow RM, Attonito JM, et al. Posttraumatic stress disorder symptomatology and alcohol use among HIV-seropositive adults in Haiti. AIDS Care. 2013;25(10):1210-1218. doi:10.1080/ 09540121.2013.763894

49. Nygaard E, Heir T. World assumptions, posttraumatic stress and quality of life after a natural disaster: a longitudinal study. Health Qual Life Outcomes. 2012;10(1):76. doi:10.1186/1477-7525-10-76

50. Ozdin S, Bayrak Ozdin S. Levels and predictors of anxiety, depression and health anxiety during COVID-19 pandemic in Turkish society: the importance of gender. Int J Soc Psychiatry. 2020;66 (5):504-511. doi:10.1177/0020764020927051

51. Yıldırım M, Geçer E, Ömer A. The impacts of vulnerability, perceived risk, and fear on preventive behaviours against COVID-19. Psychol Health Med. 2020;1-9.

52. Olagoke AA, Olagoke OO, Ashley MH. Exposure to coronavirus news on mainstream media: the role of risk perceptions and depression. Br J Health Psychol. 2020;e12427.

53. Asmundson GJG, Taylor S. How health anxiety influences responses to viral outbreaks like COVID-19: what all decision-makers, health authorities, and health care professionals need to know. $J$ Anxiety Disord. 2020;71:102211. doi:10.1016/j.janxdis.2020.102211

54. Francis JM, Newby JM, O’Moore K, Tang S, Christensen H, Faasse K. Acute mental health responses during the COVID-19 pandemic in Australia. PLoS One. 2020;15(7):e0236562. doi:10.1371/journal.pone.0236562

55. Harper CA, Satchell LP, Fido D, Latzman RD. Functional fear predicts public health compliance in the COVID-19 pandemic. Int $J$ Ment Health Addict. 2020;1-14.
Neuropsychiatric Disease and Treatment

\section{Publish your work in this journal}

Neuropsychiatric Disease and Treatment is an international, peerreviewed journal of clinical therapeutics and pharmacology focusing on concise rapid reporting of clinical or pre-clinical studies on a range of neuropsychiatric and neurological disorders. This journal is indexed on PubMed Central, the 'PsycINFO' database and CAS, and
Dovepress

is the official journal of The International Neuropsychiatric Association (INA). The manuscript management system is completely online and includes a very quick and fair peer-review system, which is all easy to use. Visit http://www.dovepress.com/testimonials.php to read real quotes from published authors. 